\title{
Loss of Excitation Detection in Synchronous Generators Based on Dynamic State Estimation
}

\author{
Pablo Marchi, Pablo Gill Estevez, Francisco Messina, Cecilia G. Galarza
}

\begin{abstract}
In this paper, we present a new approach to the detection of Loss-Of-Excitation (LOE), a typical failure of synchronous generators. Unlike most of the algorithms proposed in the literature, which only use the information available at the point of connection, we also take advantage of prior knowledge of the generator model. To track the field voltage and the other state variables, we have chosen the Constrained Unscented Kalman Filter (CUKF) as the core estimation technique, with phasor measurements as the input for this filtering algorithm. Detection of LOE is then performed by using the Faulty Modes Detection and Diagnosis (FMDD) algorithm, which combines the normal operation and a LOE-based model to decide in real-time whether an LOE has occurred or not. Results of simulations using a small two-area power system and the IEEE 39-bus system show that LOE detection times can be significantly reduced as compared to conventional and state-of-the-art approaches. Moreover, we observe that the new fault detection signal used to trip the generator can avoid short-term voltage stability problems.
\end{abstract}

Index Terms-Loss of excitation, synchronous generators, fault detection and diagnosis, generator modeling, PMU data, constrained unscented Kalman filter.

\section{INTRODUCTION}

$\mathbf{T}$ HE excitation system plays a fundamental role in synchronous generators since it provides the field voltage necessary to maintain the synchronism between the machine and the rest of the power system. Unfortunately, the excitation system is not fail-safe. Indeed, several types of faults can occur in the excitation system, such as field short circuits, field open circuits, accidental tripping of field breaker, loss of supply of the main exciter, poor brush contact in the exciter, field circuit breaker latch failure, voltage regulation system failures and slip ring flashovers [1]. When any of these faults take place, the generator starts to behave as an asynchronous generator and begins to absorb reactive power from the network while the speed of the rotor increases. This can lead to high stator winding currents, severe pulsating torque, stator end-core overheating and heavy thermal heating in the rotor body [2]. Usually, the rotor gets damaged in the process. Moreover, this behavior can trigger voltage stability problems in the power system leading to major system disturbances and blackouts. To

The work of P. Marchi was supported by a Peruilh Ph.D. grant. This work was partially funded by the UREE4 FONARSEC project "Development of Synchrophasor Measurements for Smart Electrical Grids", funded by the Ministry of Science, Technology and Innovation of Argentina, and the PUE 22920160100117CO project: "Characterization of advanced electrical networks using numerical modeling and distributed sensing" funded by the CONICET.

P. Marchi, P. Gill Estevez and C. G. Galarza are with the CSC-CONICET, and the School of Engineering, Universidad de Buenos Aires, Argentina. F. Messina works with the department of Electrical and Computer Engineering, McGill University, Canada. (e-mail: pmarchi@fi.uba.ar, pgill@ fi.uba.ar, fmessina@fi.uba.ar, cgalar@fi.uba.ar). avoid all these possible issues, loss-of-excitation (LOE) must be detected and treated as soon as possible. The importance of LOE protection has been stressed in [3] and [4].

Nowadays, guides and tutorials about the protection of synchronous generators have been released by the IEEE Power System Relaying Committee [1], [5]. These publications present a review of protections against faults and abnormal operating conditions using relays to trip the generator out of the power system. Here, LOE protection mechanisms are identified as ANSI 40 to standardize the commercial equipments in the field. Besides, these devices can adopt different protection criteria based on stator impedance, reactive power, rotor voltage and bus bar voltage. Indeed, these protection schemes can be optionally combined to meet various requirements for different generators.

Some pioneers as Mason [6] and Berdy [7] introduced the use of different offset MHO relays for detecting LOE issues in synchronous generators. It is worth mentioning that these protection schemes are still being used for LOE protection in commercial equipments [8]. Even though this relay is widely used in industry, it is susceptible to malfunctioning when system disturbances, such as stable power swings and out of step conditions, are present. An important example of this issue occurred in the North American blackout of August 2003 [9], in which thirteen LOE relay errors were identified. Besides, with the increased penetration of induction motor loads (e.g., air-conditioners), power systems tend to be more vulnerable to short-term voltage instability problems [10].

Therefore, new approaches to detect LOE conditions have been developed to enhance the robustness against external faults and to decrease the time required by the relay to disconnect the generator. For example, in [11] a fuzzy-logic based method capable of reducing the trip time and showing robustness against stable power swings is presented. Unfortunately, the performance of the method depends strongly on some design parameters, and the trip times may be longer than expected when the generator is under-loaded. Then, other methodologies emerged, as the decision tree criteria [12], showing better results than other classifiers such as artificial neural networks [13]. After that, analysis using different lossof-excitation indexes (LOEI) were adopted by [14], [15], [16], showing that the trip times could be reduced even more than in previous works. The authors in [17] and [18] proposed new approaches that use the rate of change of resistance and the rate of change of angle for the classification criteria, respectively. Although these methodologies have shown good results, they are conditioned by the oscillatory frequencies that may exist in any power system. Recently, dynamic state estimation [19] and fault classification techniques have been applied in the 
context of LOE detection. In particular, the authors in [20] used two different models for normal and faulty operation. The latter models the failure in the exciter rotator diode as a gain change, and the field voltage is considered an unknown input. For that reason, the mentioned methodology is more difficult to expand for the case of multiple faults, and to use it for fault classification.

In this paper, we present a novel approach to solve the LOE problem by using dynamic state estimation (DSE) of the internal states of a generator unit and applying the Faulty Modes Detection and Diagnosis (FMDD) algorithm [21]. In particular, the model for faulty operation nulls out the excitation voltage, allowing for general fault detection and classification schemes. Moreover, we incorporate the following key issues:

- A priori knowledge of the operation modes,

- A feedback loop for the state vectors,

- Constraints in some state variables.

It is important to mention that our proposal does not replace conventional protection mechanisms, such as the interlock between the field breaker and the power breaker. Indeed, the trip signal generated by this method can be used jointly with other protection mechanisms to combine the advantages of all of them. As our main contribution, we have shown that this methodology reduces even more the disconnection time without being noticeably modified by the initial conditions of the loss-of-excitation event or the oscillatory frequencies that different disturbances can generate. Besides, we have included an analysis of the possible false alarms due to external faults, bad data, frequency instability cases, and other different initial conditions for the LOE events that were not considered in previous works.

The rest of this paper is organized as follows: In Section II, we present the models to be used by the FMDD algorithm to dynamically estimate the synchronous generator in all the scenarios shown. After that, the methodology to be implemented is briefly described in Section III, for introducing the reader to the terminology. Following, all the numerical results are presented in Section IV. Simulations of different scenarios, which include loss of excitation phenomena, external faults, bad data, short term stability problems, and frequency instability swings are presented. Then, we discuss the relevance of our work and the limitations of our findings in Section V. Finally, we elaborate on the conclusions in Section VI.

\section{SynCHRONOUS GENERATOR MODELING}

To introduce the subject, Fig. 1 shows the general structure of a power plant and its protection scheme. The system consists of a synchronous generator, a turbine governor (TG), an automatic voltage regulator (AVR), and a power system stabilizer (PSS). Here, measurements from a PMU at the point of connection will be used as inputs to the filtering algorithm. This type of technique is widely used and is known as event playback [22]. Additionally, the PMU provides phase information and better quality in the measurements.

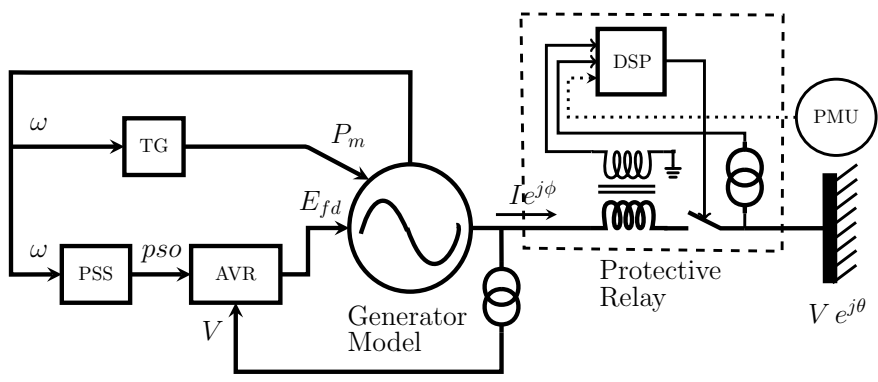

Fig. 1. Generator, relay with a digital signal processor (DSP), and PMU connected to the terminals.

To estimate the dynamics of these systems, the general form of a discrete-time state-space model for nonlinear systems is considered:

$$
\begin{aligned}
& \mathbf{x}_{k}=f_{s}\left(\mathbf{x}_{k-1}, \mathbf{u}_{k-1}\right)+\mathbf{w}_{k}, \\
& \mathbf{z}_{k}=h_{s}\left(\mathbf{x}_{k}, \mathbf{u}_{k}\right)+\mathbf{v}_{k},
\end{aligned}
$$

where $f_{s}$ is the state transition function which models the generators dynamics, $\mathbf{x} \in \mathbb{R}^{n \times 1}$ is the state vector, $\mathbf{z} \in \mathbb{R}^{m \times 1}$ is the measurement vector, $\mathbf{u} \in \mathbb{R}^{l \times 1}$ is the input vector, $h_{s}$ is the function which relates the measurements with the state vector, and $\mathbf{w} \in \mathbb{R}^{n \times 1}$ and $\mathbf{v} \in \mathbb{R}^{m \times 1}$ are noise vectors introduced to account for modeling errors.

The transient model is the simplest model that allows to add control loops into the mechanical power and field voltage. Because of that, it is widely used [23]-[25]. However, to correctly represent some external and internal faults, as the LOE event, the sub-transient effects should be considered. So, all the effects modeled by the conventional round rotor model (GENROU) will be taken into account [26]. Equations (2a) to (4a) define the behavior of this type of generator.

\section{Synchronous generator GENROU model:}

$$
\begin{aligned}
\frac{d \delta}{d t} & =\omega_{s}\left(\omega-\omega_{0}\right), \\
\frac{d \omega}{d t} & =\frac{\omega_{0}}{2 H}\left[\frac{T_{m}-D\left(\omega-\omega_{0}\right)}{\omega}-T_{e}\right], \\
\frac{d \Psi_{q}^{\prime \prime}}{d t} & =\frac{-\Psi_{q}^{\prime \prime}+K_{1} I_{q}+E_{d}^{\prime}}{T_{q}^{\prime \prime}}, \\
\frac{d \Psi_{d}^{\prime \prime}}{d t} & =\frac{-\Psi_{d}^{\prime \prime}-K_{2} I_{d}+E_{q}^{\prime}}{T_{d}^{\prime \prime}}, \\
\frac{d E_{q}^{\prime}}{d t} & =\frac{E_{f d}-\left[E_{q}^{\prime}+K_{3}\left(I_{d}+K_{4} \frac{d \Psi_{d}^{\prime \prime}}{d t} T_{d}^{\prime \prime}\right)+S_{q}\right]}{T_{d}^{\prime}}, \\
\frac{d E_{d}^{\prime}}{d t} & =\frac{-E_{d}^{\prime}+K_{5}\left(I_{q}-K_{6} \frac{d \Psi_{q}^{\prime \prime}}{d t} T_{q}^{\prime \prime}+S_{d}\right)}{T_{q}^{\prime}},
\end{aligned}
$$

Automatic voltage regulator model:

$$
\begin{aligned}
& \frac{d V_{T R}}{d t}=\frac{1}{T_{R}}\left(V-V_{T R}\right), \\
& \frac{d E_{f d}}{d t}=\frac{1}{T_{A}}\left[-E_{f d}+K_{A}\left(p s o+V_{R E F}-V_{T R}\right)\right],
\end{aligned}
$$

Turbine governor model:

$$
\frac{d T_{m}}{d t}=\frac{1}{T_{e f}}\left[-T_{m}+\left(\omega_{0}-\omega\right) \frac{1}{r}+T_{m 0}\right] .
$$




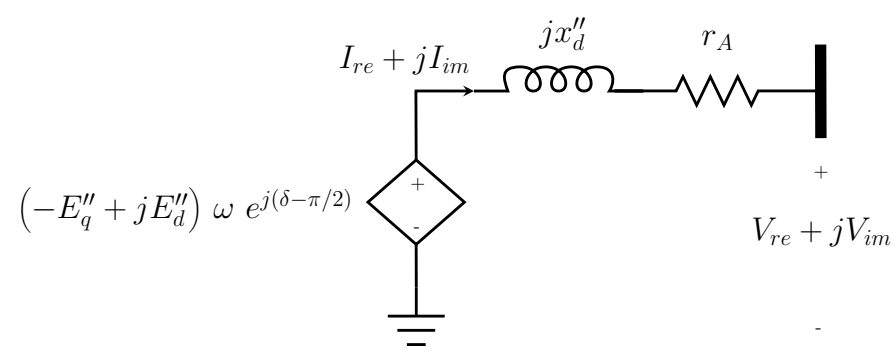

Fig. 2. Synchronous machine sub-transient dynamic circuit model.

To complete the system, additional algebraic equations and constants are given in Appendix A. A full description of the notation is given in Appendix B. The model presented above can be found in [27] and will be used by the filtering algorithm to obtain the results of Section IV. The saturation functions present in (2e) and (2f) will not be incorporated. To simplify the exposition, the model for the PSS has not been implemented. Alternatively, its output could be included in (3b) as pso. Therefore, the state vector is defined as:

$$
\mathbf{x}=\left[\begin{array}{lllllllll}
\delta & \omega & \Psi_{q}^{\prime \prime} & \Psi_{d}^{\prime \prime} & E_{q}^{\prime} & E_{d}^{\prime} & V_{T R} & E_{f d} & T_{m}
\end{array}\right]^{T} .
$$

Using the same criterion as in [28], the measurement vector is composed of the real and imaginary parts of the voltage phasor at the point of connection:

$$
\mathbf{z}=\left[V_{r e} V_{i m}\right]^{T} .
$$

Under this criterion, $\mathbf{z}_{k}$ will contain an associated noise whose distribution corresponds to the distribution of the error in the voltage phasor measurement. To relate these measurements to the state vector, and assuming that $x_{q}^{\prime \prime} \approx x_{d}^{\prime \prime}$, the electrical interface shown in Fig. 2 is used. After some algebraic manipulations, the measurements equations result:

$$
\begin{aligned}
V_{r e} & =\left[-E_{q}^{\prime \prime} \sin (\delta)+E_{d}^{\prime \prime} \cos (\delta)\right] \omega-I_{r e} r_{A}+I_{i m} x_{d}^{\prime \prime}, \\
V_{i m} & =\left[E_{q}^{\prime \prime} \cos (\delta)+E_{d}^{\prime \prime} \sin (\delta)\right] \omega-I_{i m} r_{A}-I_{r e} x_{d}^{\prime \prime}
\end{aligned}
$$

At last, the control vector necessary to complete the model of the generator is composed by the current signals:

$$
\mathbf{u}=\left[\begin{array}{ll}
I_{\text {re }} & I_{i m}
\end{array}\right]^{T} .
$$

These signals are obtained from the current channel of the PMU device.

\section{Methodology}

To detect the LOE problem, we propose to use an interacting multiple-model estimation algorithm for fault detection and diagnosis (FMDD). The FMDD algorithm is presented in [21] and it was initially used for fault detection in aircraft and satellites. Such an algorithm is based on an interacting multiple-model estimation method. The basic idea is to use several Kalman filters simultaneously and to combine the estimation of each of them to create a valid update of the state vector. In turn, the algorithm is one of the most cost-effective estimation techniques for systems involving structural as well as parametric changes [21].

Given its simplicity, its reduced computational cost, and its good performance for non-linear systems, we have adopted the Constrained Unscented Kalman Filter (CUKF) as the Kalman filter to be used. Indeed, as it was shown in [23], the Unscented Kalman Filter (UKF) is a feasible real-time solution. As compared with the extended Kalman filter, it should be noted that the UKF involves a more accurate higherorder approximation for the nonlinear functions $f_{s}$ and $h_{s}$. Besides, it does not require the computation of the Jacobian of these functions. This algorithm defines the unscented transformation to approximate the mean $\hat{\mathbf{x}}_{k}$ and covariance $\hat{\mathbf{P}}_{k}$ of the state vector. For this purpose, the concept of sigma points is introduced. These sigma points are propagated through the nonlinear functions and then they are constrained by the projection operator $\mathcal{P}$ to restrict them to the feasible region of the state-space. In this case, the operator is defined as a saturation based on the generator model parameters. For example, the $E_{f d}$ and $T_{m}$ variables will be constrained to the intervals $\left[V_{R \max }, V_{R \min }\right]$ and $\left[T_{m \max }, T_{m \min }\right]$, respectively, when the computation of the sigma points is made. Then, the mean and the covariance for $\mathbf{x}_{k}$ and $\mathbf{z}_{k}$ are approximated using a weighted sample mean and covariance of the posterior sigma points. The procedure can be divided into two stages: prediction and correction. For an in-depth discussion of the CUKF algorithm, the reader is referred to [29].

Under the FMDD approach, the user defines an a priori transition probability matrix which sets up the probabilities to switch from the actual mode of operation to the others. After that, the a posteriori probability is computed using the value of the innovations of each filter. The key quantities here are the innovations $\mathbf{y}_{k}$. If a match between the measurements and the simulated model occurs, after convergence of the Kalman filter, the innovations are expected to be concentrated around zero with a covariance matrix given by $\mathbf{S}_{k}$. In contrast, if a different model is used (e.g., normal operation model while LOE has occurred), this will no longer be valid. In other words, the innovations should be a good indicator of the likelihood that a particular model is a correct representation of the phenomenon being observed. Hence, this approach is used when the faulty models are accurate enough to represent the system faults.

\section{FMDD Algorithm}

The main goal of the FMDD algorithm is to identify which one of the operation modes $m^{j}$ is the one which represents the system dynamics at each instantaneous discrete time $k$. Indeed, each operation mode of the system (normal or faulty) is linked to a mathematical model defined by a set of transition functions $\mathcal{M}=\left\{f_{s 1}, f_{s 2}, \cdots, f_{s N}\right\}$, and it evolves according to an a priori transition probability matrix $\Pi \in \mathbb{R}^{N \times N}$, where each element is defined by (9) below. The proper choice of this matrix is based on the expected sojourn time of each mode [21]. The principal assumption of this approach is that the nonlinear model system dynamics (1) can be accurately approximated by a first-order "Markov hybrid nonlinear system" [21]. The complete algorithm is shown in Fig. 3. As it can be observed, it consists of several stages which are described below.

$$
\boldsymbol{\Pi}_{i j}=P\left(m_{k+1}^{j} \mid m_{k}^{i}\right), i, j=1, \ldots, N .
$$




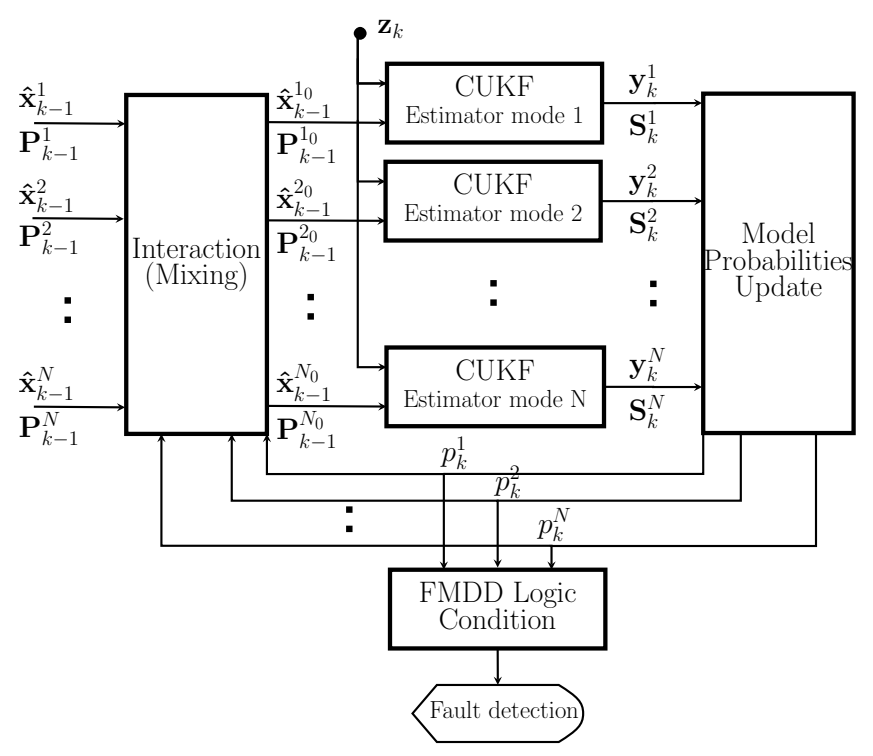

Fig. 3. Block diagram of the FMDD algorithm.

Stage 1 - Interaction (Mixing): Computes the input state vector and covariance matrix of each Kalman filter. The main goal of this stage is to ensure the Kalman filter convergence for all the estimator modes of operation. Hence, a linear combination of all the state vectors $\hat{\mathbf{x}}^{j}$ is implemented. In other words, the input of each Kalman filter at $k-1$ is modified by pondering each one of the state vectors and covariances output using the a posteriori probabilities $p_{k}^{j}=P\left(m_{k}^{j} \mid \mathbf{y}_{k}^{j}, \mathbf{S}_{k}^{j}\right)$ of the operation mode $m^{j}$. These probabilities and the a posteriori transition probabilities $\boldsymbol{M}_{k}^{i j}=P\left(m_{k}^{i} \mid m_{k-1}^{j}, \mathbf{y}_{k}^{j}, \mathbf{S}_{k}^{j}\right)$ are computed as follows:

$$
\begin{array}{rlrl}
p_{k \mid k-1}^{j} & =\sum_{i=1}^{N} \boldsymbol{\Pi}_{i j} p_{k-1}^{i} & j=1, \ldots, N \\
\boldsymbol{M}_{k}^{i j} & =\frac{\boldsymbol{\Pi}_{i j} p_{k-1}^{i}}{p_{k \mid k-1}^{j}} & i, j=1, \ldots, N .
\end{array}
$$

Here, the innovation vectors of each mode $\mathbf{y}_{k}^{j}$ and the innovation covariances $\mathbf{S}_{k}^{j}$ are taken as observations in the FMDD algorithm feedback. Then, the input state vector $\hat{\mathbf{x}}_{k}^{j_{0}}$ and its respective covariance matrix $\mathbf{P}_{k}^{j_{0}}$ is computed for each one of the filters as a linear combination of its outputs as:

$$
\begin{aligned}
\hat{\mathbf{x}}_{k-1}^{j_{0}} & =\sum_{i=1}^{N} \hat{\mathbf{x}}_{k-1}^{i} \hat{\mathbf{M}}_{k}^{i j}, \quad j=1, \ldots, N, \\
\mathbf{P}_{k-1}^{j_{0}} & =\sum_{i=1}^{N}\left[\mathbf{P}_{k-1}^{i}+\left(\hat{\mathbf{x}}_{k-1}^{j_{0}}-\hat{\mathbf{x}}_{k-1}^{i}\right)\right. \\
& \left.\ldots \quad\left(\hat{\mathbf{x}}_{k-1}^{j_{0}}-\hat{\mathbf{x}}_{k-1}^{i}\right)^{T}\right] \hat{\mathbf{M}}_{k}^{i j} .
\end{aligned}
$$

Stage 2 - Estimates update: Each of the state estimates of the models included in the set $\mathcal{M}$ is updated one time step (from $k-1$ to $k$ ), using the Kalman filtering technique.
Stage 3 - Compute the a posteriori probabilities: This stage is performed using the Bayes formula:

$$
p_{k}^{j}=\frac{\mathcal{L}_{k}^{j} p_{k \mid k-1}^{j}}{\sum_{i=1}^{N} \mathcal{L}_{k}^{i} p_{k \mid k-1}^{i}}, \quad j=1, \ldots, N,
$$

where $\mathcal{L}_{k}^{j}=P\left(\mathbf{y}_{k}^{j} \mid m_{k}^{j}, \mathbf{S}_{k}^{j}\right)$ represents the likelihood function. As it is assumed that the innovations have a Gaussian distribution with zero mean and covariance matrix $\mathbf{S}_{k}^{j}, j=1, \ldots, N$, the likelihood function can be computed as: $\mathcal{L}_{k}^{j}=\left|2 \pi \mathbf{S}_{k}^{j}\right|^{-1 / 2} \exp \left(-\frac{1}{2} \mathbf{y}_{k}^{j} \mathbf{S}_{k}^{j-1} \mathbf{y}_{k}^{j}\right)$.

Stage 4 - Fault detection: The fault detection criterion is defined by the comparison between the maximum value of all the probabilities of the failure operation modes $p_{F M D D \max }=\max \left\{p_{k}^{j}\right\}, j=2, \ldots, N(j=1$ is assigned to the normal operation mode) and a predefined threshold:

$$
\text { If : } p_{F M D D \max }>p_{T h r}, \Rightarrow \text { Fault detected. }
$$

Besides, in general, the origin of the fault can be identified using the $j$ index. Henceforward, we consider that the only failure modeled is the LOE fault. Notice that the two models considered here can be defined changing the equations of the transition functions as follows:

$$
\begin{aligned}
& f_{s 1} \rightarrow \text { Eqs. }(2 \mathrm{a})-(4 \mathrm{a}), \\
& f_{s 2} \rightarrow \text { Eqs. }(2 \mathrm{a})-(2 \mathrm{f}),(4 \mathrm{a}),\left(E_{f d}=0\right) .
\end{aligned}
$$

Nevertheless, other types of failures can be easily incorporated using this methodology and extend its use for other applications, as long as they can be modeled using a state-space representation. For example, field circuit conditions during various LOE failures, as modeled in [30], could be included.

\section{NUMERICAL RESULTS}

The simulated measurements of the PMU are generated using both, Power System Toolbox (PST) [31] and PSS/E. Different scenarios are simulated. The PST software is used for a two-area power system, while the PSS/E is used to simulate an IEEE 39 bus system. The simulated measurements for each scenario include the voltage and current phasors, and they are generated considering a reporting rate of $f_{r}=60 \mathrm{fps}$ and additive white Gaussian noise (AWGN). The time between samples is set to $T_{s}=1 /\left(f_{r} . n_{\text {cycle }}\right)$, where $n_{\text {cycle }}=12$ is the number of samples per cycle. Here, we are considering that the PMU can transmit the phasor measurement data almost at its sample rate. This is not a far-fetched assumption since it is assumed that the PMU has a point to point link with the relay. In this context, the average computing time for each PMU frame of the FMDD algorithm is $6.8 \mathrm{~ms}$. All the tests were performed on a PC with Intel ${ }^{\circledR}$ Core $^{\mathrm{TM}}$ i7-3770 CPU @ 3.40GHz × 8, 16GB DDR3 RAM: 1333 MT/s. We can conclude that the proposed method have computing times lower than the actual PMU report rate, which is $16.7 \mathrm{~ms}$ for 60 fps. Thus, the algorithm is suitable for real-time applications.

The variance of the noise is adjusted based on the following criterion for the total vector error (TVE) standard metric: $\mathrm{TVE}=1 \%$. Concretely, the voltage noise is modeled as 


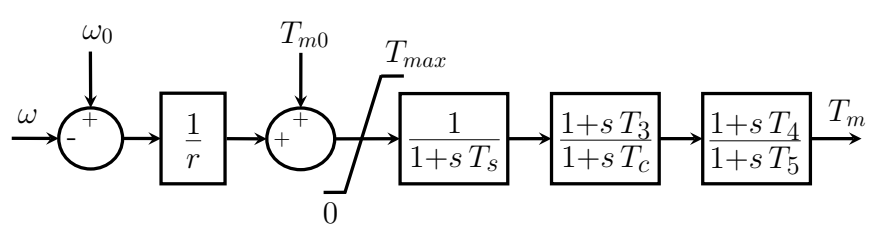

Fig. 4. Turbine-governor model for the two-area power system.

a complex and circularly-symmetric random variable, i.e, $\sigma_{V_{r e}}=\sigma_{V_{i m}}=|\vec{V}| \mathrm{TVE} /(3 \sqrt{2})$, and assuming $|\vec{V}| \approx 1$ p.u.

The results presented below were obtained by proposing an arbitrary initial condition including a 10\% deviation from the true state variables. As the initial condition modifies the convergence time of the initialization process, we have chosen a reasonable value that does not increase too much the simulation time needed for all the tests. Besides, the parameters for the CUKF were selected using the same criteria as in [32]. The following a priori transition probability matrix for all the scenarios is assumed:

$$
\boldsymbol{\Pi}=\left[\begin{array}{cc}
10^{-8} & 1-10^{-8} \\
1-10^{-10} & 10^{-10}
\end{array}\right] .
$$

The results shown in this paper are compared against [7] and [17]. In [7], the equivalent impedance of the network $Z_{e q}=R_{e q}+j X_{e q}$ (i.e., $\left.Z_{e q}=\left(V_{r e}+j V_{i m}\right) /\left(I_{r e}+j I_{i m}\right)\right)$ is used to establish the disconnection criteria. This methodology proposes to define two regions of action in the complex impedance plane, called Zone 1 and Zone 2 respectively. The Zone 1 is a circle of diameter 1 p.u and centered at the point $-j\left(x_{d}^{\prime}+1\right) / 2$ [6]. If the equivalent impedance calculated by the relay $Z_{e q}$ reaches Zone 1 , the disconnection of the generator units should be done instantly. Also, the relay will then be activated if the equivalent impedance computed by the relay enters into Zone 2 and remains inside for more than $0.3 s$ [7]. On the other hand, in [17] the sign of the rate of change of resistance $d R_{e q} / d t$ is used to define a setting-free approach. To distinguish an LOE event from the slowest power swings, the method defines that $d R_{e q} / d t$ must remain negative for more than $1.7 \mathrm{~s}$.

First, we consider the classical two-area and four machine system shown in Fig. 5. The PST is used to perform the simulations. The parameters for the lines and buses can be found in the example file $d 2$ asbeg.m. Here, the apparent power base is set to $S_{\text {base }}=100$ MVA. A sub-transient generator model for all generators is assumed with a ESAC4A [26] as the excitation system and a turbine governor as shown in Fig. 4. Besides, all the loads are modeled as conventional PQ buses. The parameters of the sub-transient generator model, as well as the AVR and TG systems, can be found in Tables IV and V in Appendix C. Note that the base for all the generators is set to $900 \mathrm{MVA}$ and saturation effects of the core are considered.

For this power system, all the tested scenarios are listed below. The scenarios fall into three categories: (A) LOE events, (B) external fault events, and (C) bad data. On the one hand, we prove that for the proposed methodology all the cases arising after an LOE event are detected. On the other hand, we verify that external faults do not produce false alarms. For

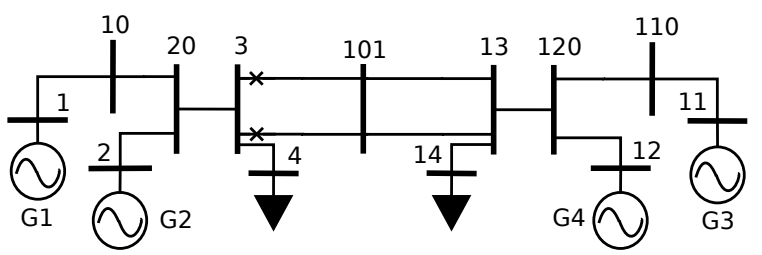

Fig. 5. Single line diagram of the two-area power system.

TABLE I

MeAn AND STANDARD DEVIATION OF THE DETECTION TIMES $\left(\hat{\mu}_{t_{d}}, \hat{\sigma}_{t_{d}}\right)$ AFTER 1000 MONTE-CARLO TRIALS.

\begin{tabular}{|c|c|c|c|c|c|c|}
\cline { 2 - 7 } \multicolumn{1}{c|}{} & \multicolumn{3}{c|}{$\hat{\mu}_{t_{d}}[s]$} & \multicolumn{3}{c|}{$\hat{\sigma}_{t_{d}}[m s]$} \\
\hline Scenarios & {$[7]$} & {$[17]$} & FMDD & {$[7]$} & {$[17]$} & FMDD \\
\hline A1 & 2.88 & 2.17 & 0.44 & 4.02 & 8.97 & 12.81 \\
\hline A2 & 4.23 & 2.15 & 0.51 & 6.24 & 10.30 & 17.02 \\
\hline A3 & 3.36 & 2.17 & 0.48 & 4.91 & 10.21 & 14.65 \\
\hline A4 & 4.70 & 2.36 & 0.61 & 2.28 & 376.5 & 32.49 \\
\hline
\end{tabular}

all the scenarios, the system remains in a stationary state for 2 seconds and then a fault is applied.

\section{A. Two-area power system: Loss of excitation.}

A1: Heavily loaded generator with lagging power factor $(\mathrm{PF}=0.9746)$. The initial active and reactive power of the generator G1 is set to $\left(p_{G 1}=7.00\right.$ p.u. and $q_{G 1}=1.61$ p.u.) respectively.

A2: $\quad$ Lightly loaded generator with lagging $\mathrm{PF}=0.9277$ ( $p_{G 1}=4.00$ p.u. and $q_{G 1}=1.61$ p.u.).

A3: Average loaded generator with lagging $\mathrm{PF}=0.9519$ $\left(p_{G 1}=5.00\right.$ p.u. and $q_{G 1}=1.61$ p.u. $)$.

A4: Heavily loaded generator with partial LOE. Identical to Scenario A1 with the exception that the field voltage is reduced $50 \%$.

To understand the differences between these scenarios, Fig. 6 shows the equivalent impedance $Z_{e q}$ seen from the relay terminals. Here, the LOE fault generates a change in the field voltage of the generator G1 to zero except for the scenario A4. As it can be seen, A1 and A4 are very similar. The difference between them is the time in which the equivalent impedance reaches the relay action zones. For Scenario A2, it can be distinguished that the relay gets triggered because the time in Zone 2 was exceeded. Fig. 7 shows the results of applying the FMDD algorithm for all the cases. As can be observed, the probability of the failure mode $p^{2}$ exceeds the threshold $p_{T h r}$ after less than one second from the moment that the fault takes place for all cases. In spite of the mismatch between the failure model and the real simulation, the scenario A4 has shown a good performance, thus showing that the proposed method is robust. Also, a Monte-Carlo analysis has been made to guarantee that the noise of the measurements does not affect the quality of the detections. The results are shown in Table I, where we have also included the results using the Berdy criterion and the method proposed in [17] for comparison purposes. Note that the disconnection times are highly reduced in the four different scenarios. 


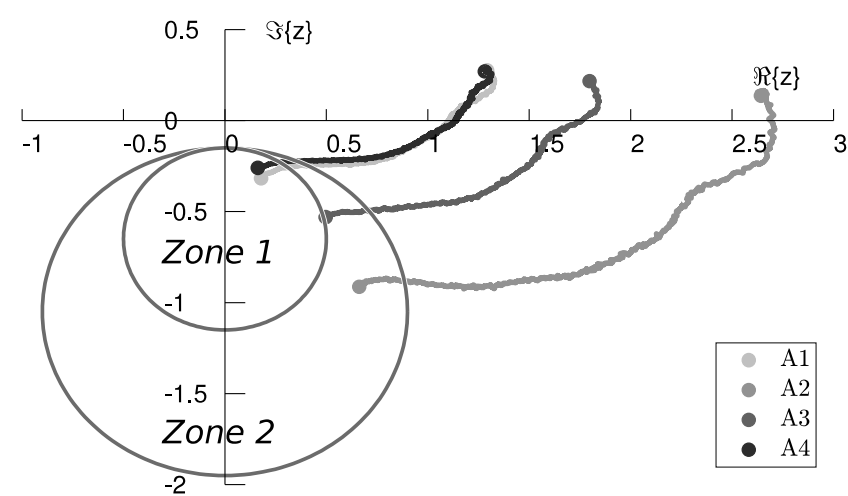

Fig. 6. Scenario A: Evolution of the equivalent impedance $Z_{e q}$ in the impedance plane for loss of excitation events.

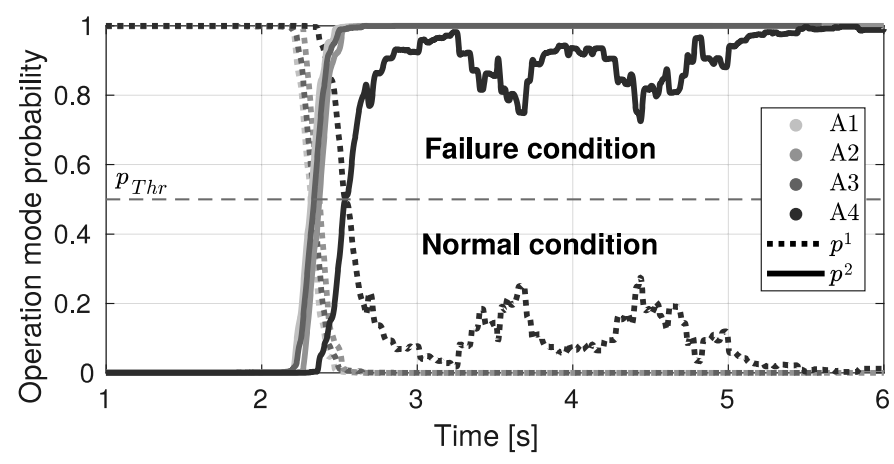

Fig. 7. Scenario A: Operation mode probabilities $p^{1}$ and $p^{2}$ as a function of time for loss of excitation events.

\section{B. Two-area power system: External faults.}

B1: Three-phase to ground short circuit. The fault is applied in bus number 3. It remains for $133 \mathrm{~ms}$ and then it is cleared.

B2: Single line to ground short circuit. The fault is applied in bus number 3. It remains for $200 \mathrm{~ms}$ and then it is cleared.

B3: Loss of line with no fault. One of the line between the nodes 101 and 13 is lost and can not be reconnected.

B4: Loss of load at bus 4 . The load in bus number 4 is disconnected.

For these scenarios, the probabilities of normal and failure operation are shown in Fig. 8. The results show that the probability of malfunction does not exceed the threshold for any of the simulated scenarios. The disconnection of the generator does not occur even when performing a Monte Carlo analysis, in any of the scenarios and trials made. Analyzing the results, it can be concluded that false alarms could be generated after severe and prolonged faults such as a threephase to ground. Nevertheless, in such cases, other external protections are more likely to act before the LOE protection.

Finally, it can be concluded that there is a trade-off between the disconnection time and the probability of false alarm depending on the threshold $p_{T h r}$. For this reason, a receiver operation characteristic was obtained after 1000 Monte Carlo trials were performed, considering the scenario A4 as the positive condition and $\mathrm{B} 1$ as the negative condition. The results

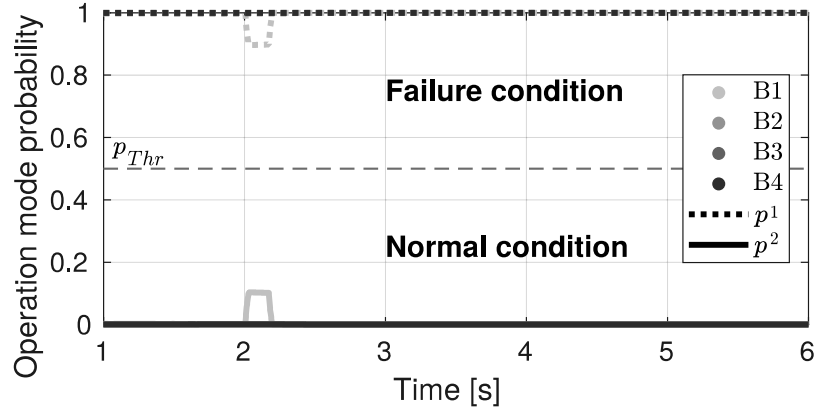

Fig. 8. Scenario B: Operation mode probabilities $\hat{\mu}_{1}$ and $\hat{\mu}_{2}$ as a function of time for external faults.

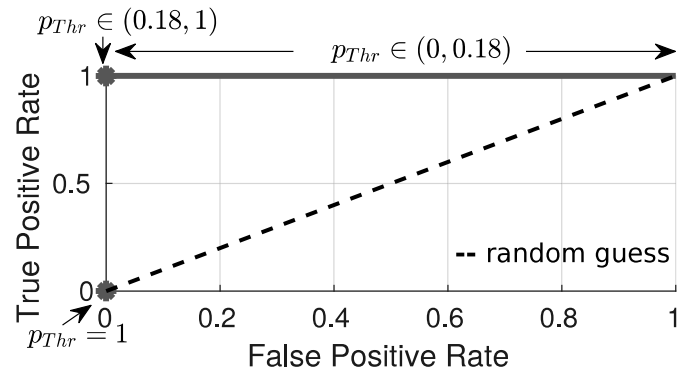

Fig. 9. Receiver operating characteristic curve with the diagnostic ability of a the binary classifier (15) as its discrimination threshold $p_{T h r}$ is varied.

can be seen in Fig. 9, and it can be concluded that values higher than $p_{T h r}>0.18$ provide a negligible false positive rate under these scenarios. To ensure us that false alarms do not occur with a certain safety margin, and to avoid prolonged trip times, we have chosen to set the threshold to $p_{T h r}=0.5$.

\section{Two-area power system: Bad data.}

In this section, we consider gross errors in the phasor measurements and show how the performance of the different methodologies is degraded. First, we consider that the errors occur in the voltage channel and arrive at different instants of time, defined as variable $t_{\text {bad }}$. We assume that the times are distributed following an exponential distribution function, i.e. $t_{\text {bad }} \sim \operatorname{Exp}\left(\lambda_{\text {bad }}=2\right)$. At these times, the real and imaginary parts of the voltage phasor change suddenly by a multiplicative noise. Considering this, the measurement signals are changed as:

$$
V_{r e}^{b a d}=V_{r e} \eta_{b a d}, \quad V_{i m}^{b a d}=V_{i m} \eta_{b a d}
$$

where $\eta_{\text {bad }} \sim \mathcal{N}(1,0.2)$. As an example, Fig. 10 shows the real and imaginary parts of the phasor voltage at the point of connection for this Scenario (C1). We repeated the Monte Carlo analysis to get the statistics of how many times the methodologies correctly detect the system failure in Scenarios A and how many false alarms are generated by the external faults of Scenarios B. The results are shown in Table II. As can be seen, the relay is the most robust method because it can detect the failure in spite of the bad data included in the measurements, and it does not generate false alarms. Besides, it can be concluded that the performance of our proposal is highly reduced, especially for Scenario A4, where a mismatch between the faulty model and the simulation exists. 


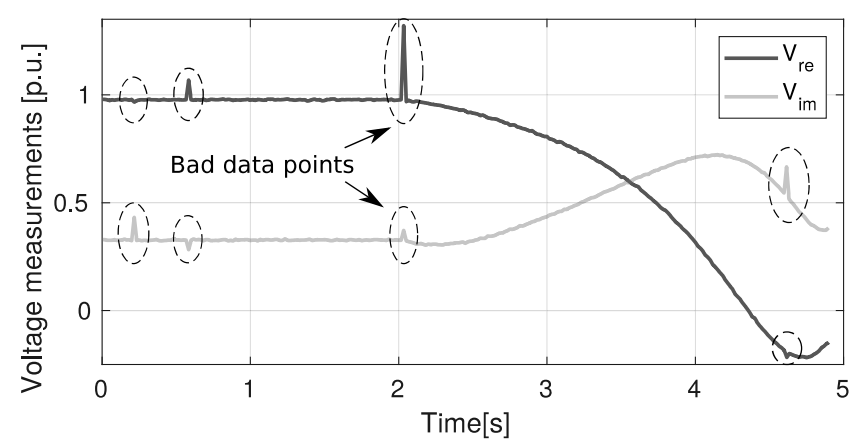

Fig. 10. Scenario C1: Voltage measurements including bad data for escenario A1.

TABLE II

Percent of times that THE LOE EVEnT IS DETECTEd AFTER 1000 MONTE-CARLO TRIALS.

\begin{tabular}{|c|c|c|c|c|c|c|}
\cline { 2 - 7 } \multicolumn{1}{c|}{} & \multicolumn{3}{c|}{ Scenario A } & \multicolumn{3}{c|}{ Scenario B } \\
\hline Sub-Scenario & {$[7]$} & {$[17]$} & FMDD & {$[7]$} & {$[17]$} & FMDD \\
\hline 1 & 100 & 77.4 & 68.6 & 0 & 0 & 8.7 \\
\hline 2 & 100 & 94.8 & 51.1 & 0 & 0 & 6.9 \\
\hline 3 & 100 & 88.9 & 57 & 0 & 0 & 7.8 \\
\hline 4 & 98.5 & 91.5 & 34.7 & 0 & 0 & 5.9 \\
\hline
\end{tabular}

On the other hand, it should be taken into account that these types of errors are very unlikely, since they can be filtered by the estimation algorithm or detected by a cyclic redundancy check in the data transmission link. For that reason, we have considered another scenario (C2) where the samples which contains errors are properly detected and discarded. If this occurs, the signal value will be assumed to be equal to the value of the last instant in which no errors were found. In such a case, none of the methods compared has demonstrated to be altered by this condition unless a burst of errors occurs. Then, we found it interesting to analyze how many consecutive bad data points would be needed to degrade the performance of the FMDD algorithm. Fig. 11 shows the true positive rate and the false positive rate as a function of the number of errors accumulated in time. We have considered the scenario A1 as the positive condition and $\mathrm{B} 4$ as the negative condition because they have shown to be more susceptible to the absence of new data. Here, the bad data condition is included in the voltage phasor after $3 \mathrm{~s}$ of simulation, when the dynamics of the system is still dominated by the LOE event. As can be observed, the methodology we propose is more likely to stop detecting LOE events than to generate false alarms. Besides, it can be concluded that the time required to decrease the performance of the method is close to $0.25 \mathrm{~s}$, which is equivalent to 15 reports of a PMU.

\section{IEEE 39-Bus System.}

In this section, a bigger and more complex system as the IEEE 39 bus system is simulated using the PSS/E software. The IEEE 39 bus system is well known as the 10-machine New-England Power System and its line diagram can be seen in Fig. 12. The models used for the generators, exciters, and

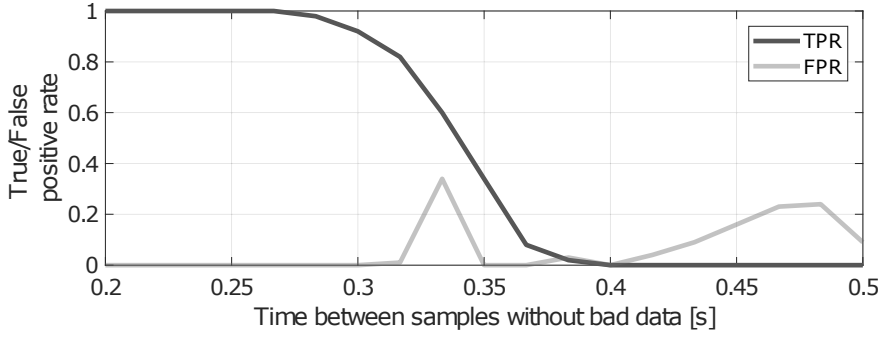

Fig. 11. Scenario C2: True/False positive rate as a function of the time between samples without bad data points.

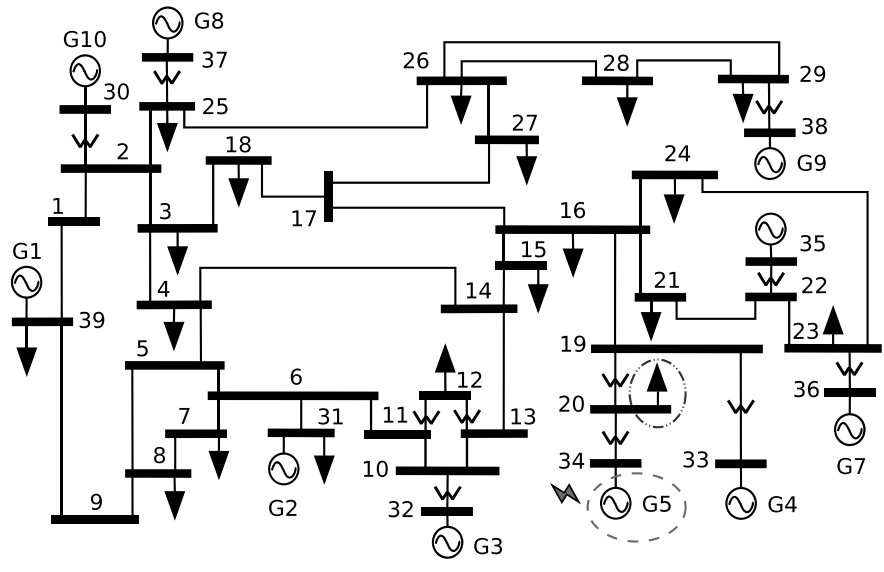

Fig. 12. IEEE 39 bus system.

turbine governor are the GENROU, ESAC4A and TGOV1 [26], respectively. The standard IEEE bus system uses conventional PQ load on all the buses. However, as the composition and utilization of end-use loads are continually evolving based on technological advances, the simulations were made using a dynamic load modeling in bus number 20 . The selection of this load was done considering the proximity to the generator affected by the LOE fault. This assumption not only proposes a more realistic scenario, but it also allows us to study short-term voltage stability problems. The structure and parameters of the load model can be found in [33]. Summarizing, these loads consist of large and small induction motors, discharge lighting, constant MVA load, and a static load response. Also included are transformer saturation effects and an equivalent distribution feeder and transformer impedance. We will consider three scenarios:

- D1 - the generator G5 is subjected to a LOE phenomenon. The field voltage drops to zero and remains constant.

- D2 - a three-phase to ground fault occurs in bus number 16 with a clearing time of $100 \mathrm{~ms}$ and loss of the line (branch 16-17).

- D3 - a three-phase to ground fault occurs in bus number 16 with a clearing time of $200 \mathrm{~ms}$ and loss of the line (branch 16-17).

The fault takes place after 1s of simulation in all scenarios. Scenario D1 was proposed to evaluate the disconnection capability of the FMDD algorithm in a more complex system. On the other hand, Scenarios D2 and D3 were proposed to ensure that the disconnection does not happen when oscillatory 

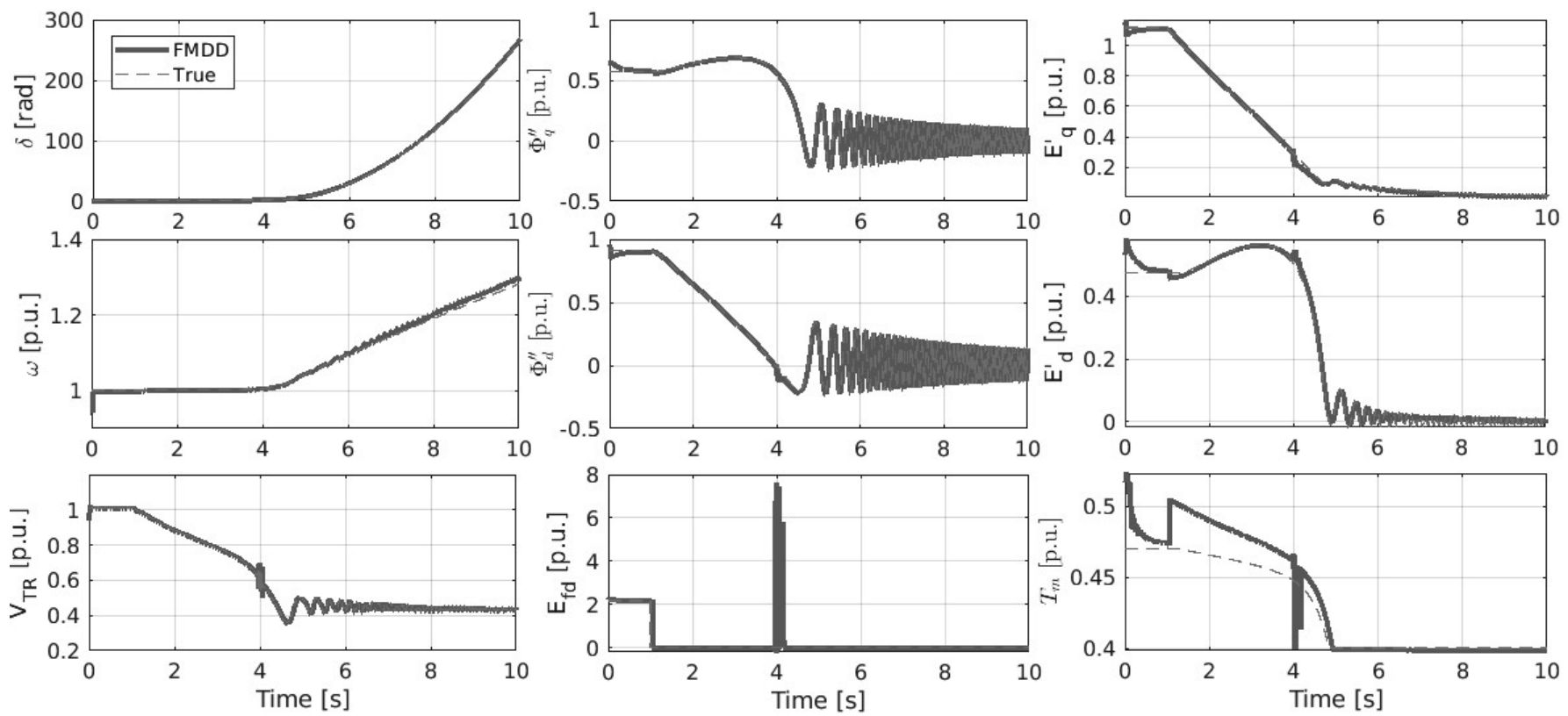

Fig. 13. Scenario D1: Global estimation of all the state variables of the generator G5 after a LOE event occurs at $1 \mathrm{~s}$.

frequencies are present at the point of connection. D2 is a scenario where stable oscillations can be observed, while instability conditions are present in D3.

\section{Scenario D1:}

Fig. 13 shows the results obtained using the proposed approach. In Fig. 13, the estimated and true state variables are shown simultaneously for the scenario D1. The global estimation of the state vector is computed as the weighted arithmetic mean using all the state vectors and the probabilities $p^{i}$ as the weights. As it can be observed, the estimates are quite close to the real behavior of the system, though the simulation considers the saturation of the magnetic core (the parameters can be found in the Appendix C) and the fact that these functions are neglected in equations (2e) and (2f). Besides, there are also some differences between the PSS/E governor model (three-pole transfer function) and the one defined by the equation (4a) (single-pole transfer function).

A Monte Carlo analysis is performed again, and the estimated means of the detection times are $\hat{\mu}_{t_{d}}=3.7 \mathrm{~s}$ for Berdy's criterion [7], $\hat{\mu}_{t_{d}}=2.93 \mathrm{~s}$ using the methodology proposed in [17], and $\hat{\mu}_{t_{d}}=372 \mathrm{~ms}$ using the FMDD algorithm in the scenario D1. It should be noted that the Berdy's criterion and the FMDD algorithm detected the failure in every single trial. Unfortunately, the algorithm proposed in [17] detected the LOE event in only $12 \%$ of the trials. Therefore, in this scenario, the disconnection using the setting-free approach [17] is not guaranteed. Fig. 14 shows what would happen to the system if the relay operates according to Berdy's criterion and it is compared against the proposed FMDD method. Note that if the relay disconnects the generator using Berdy's criterion, the voltage in the bus number 20 can be stabilized but it takes several seconds. However, the dynamic load model activates partial load shedding to return the voltage magnitude to a tolerable value. In contrast, if the FMDD algorithm is applied, the generator disconnects earlier and the system stabilizes without the need for any load disconnection.

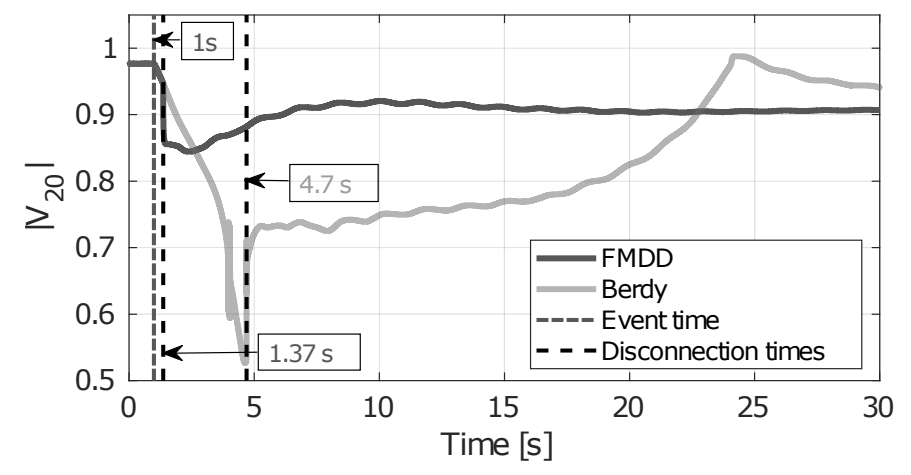

Fig. 14. Voltage magnitude in bus 20 after the disconnection of the relay.

\section{Scenarios D2 and D3:}

The simulated scenarios of this section generate frequency oscillations at the point of connection of the generator unit. These situations usually generate an inconvenience for the algorithms and methodologies oriented to detect the LOE phenomena, especially for MHO-based relays [12]. Indeed, the frequency/angular instabilities could generate false alarms and trigger the generator unit without waiting for other types of protections to act. In such a case the LOE relay malfunctions and disconnects the healthy generators which may lead to cascading blackouts. For that reason, slow and fast frequency swings are taken into account in the scenarios D2 and D3 respectively. On the one hand, Fig. 15 shows the estimated and true state variables under the effects of transient instability. The scenario D2 shows that the oscillations have a poor damping, provided by the electromechanical modes, that stabilizes the 

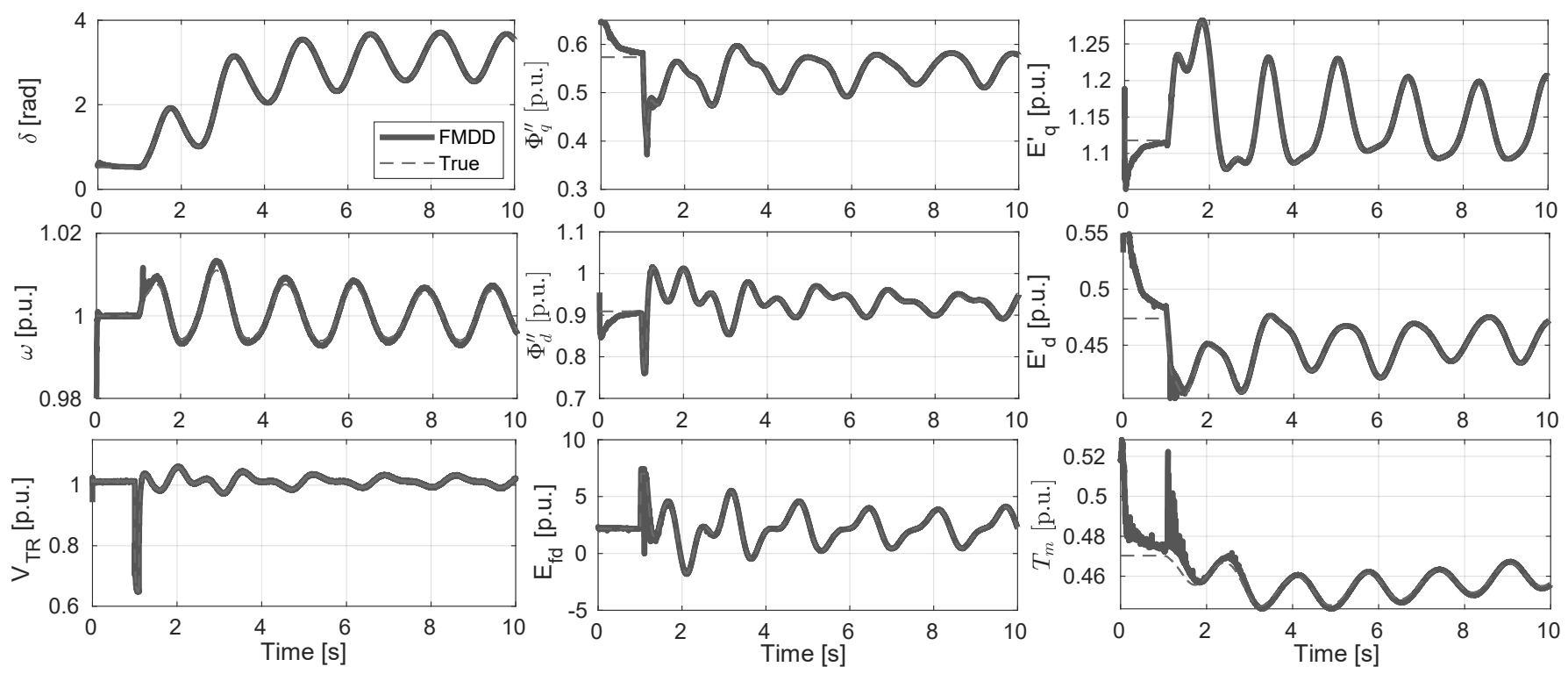

Fig. 15. Scenario D2: Global estimation of all the state variables of the generator G5 after a three-pase to ground fault with a clearing time of $100 \mathrm{~ms}$ occurs.

system to a state condition. It is worth remembering that the PSS was not modeled. On the other hand, Fig. 16 shows the estimated and true state variables after new instabilities appears at the terminals of the generator. Here, the fault simulated causes loss of synchronism of the generator. As can be seen the speed of the rotor starts with an oscillatory process and it is unable to reach the nominal frequency of the system again. A Monte Carlo analysis is performed again showing that, despite the oscillations, the FMDD algorithm does not detect a LOE condition in the generator in any of the trials made.

It is important to mention that the results presented in these scenarios were obtained when the saturation effects in the generators are neglected. Otherwise, the mismatch between the simulation and the model assumed by the CUKF filters is too large for estimating accurately the state variables, and the method cannot distinguish between a fault and a normal operating state.

\section{DISCUSSION}

Here, we have assumed a ESAC4A model as the excitation system of the generator unit. This model considers an alternator-supplied controlled-rectifier excitation system which utilizes a full thyristor bridge in the exciter output circuit. However, other excitation systems could be modeled. For example, the AC5A [26] for brushless excitation systems. But as the model becomes more complex, the number of the parameters increases, as well as the state variables. Thus, the algorithm is computationally more expensive and susceptible to the mismatch between the CUKF model and the real one. These considerations should be taken into account when the model is assigned to the generator to protect. Although we present a method that is susceptible to variations in the parameters and in the structure of the generator model, we believe that the growing development carried out in the field of generator model validation and parameter calibration make these assumptions reasonable.

Another limitations to be considered is the quality of the measurements used as an input. Particularly, we have shown that the performance of our method is highly reduced when undetected bad data is present in the phasor measurements. However, if the bad data are properly detected, we have shown that the algorithm is robust even for short bursts of bad data.

\section{CONCLUSIONS}

We have presented a new application of the FMDD algorithm to detect the loss of excitation in synchronous generators based on recent advances in dynamic state estimation. Unlike classical methodologies, which make the detection based on a set of electrical variables at the point of connection, our proposal takes advantage of the available information regarding the dynamic behavior of the generator. The results have shown that the disconnection times can be significantly reduced without producing scenarios of malfunction. Besides, as it has been shown in Section IV-D, the automatic disconnection of several loads can be avoided if the protection scheme responds fast enough.

Finally, future works could be focused on expanding the proposed method to include another types of faults, as long as these faults can be modeled in the transition function of the Kalman filter algorithm. In such settings, the classification of the fault could, in principle, be done efficiently for different faulty operation modes. However, the number and types of faults that can be successfully detected using this approach is an open question that requires further research.

\section{APPENDIX A}

\section{COMPLEMENTARY EQUATIONS}

In this appendix, we list the complementary equations of the model defined by $(2 a)-(4 a)$ : 

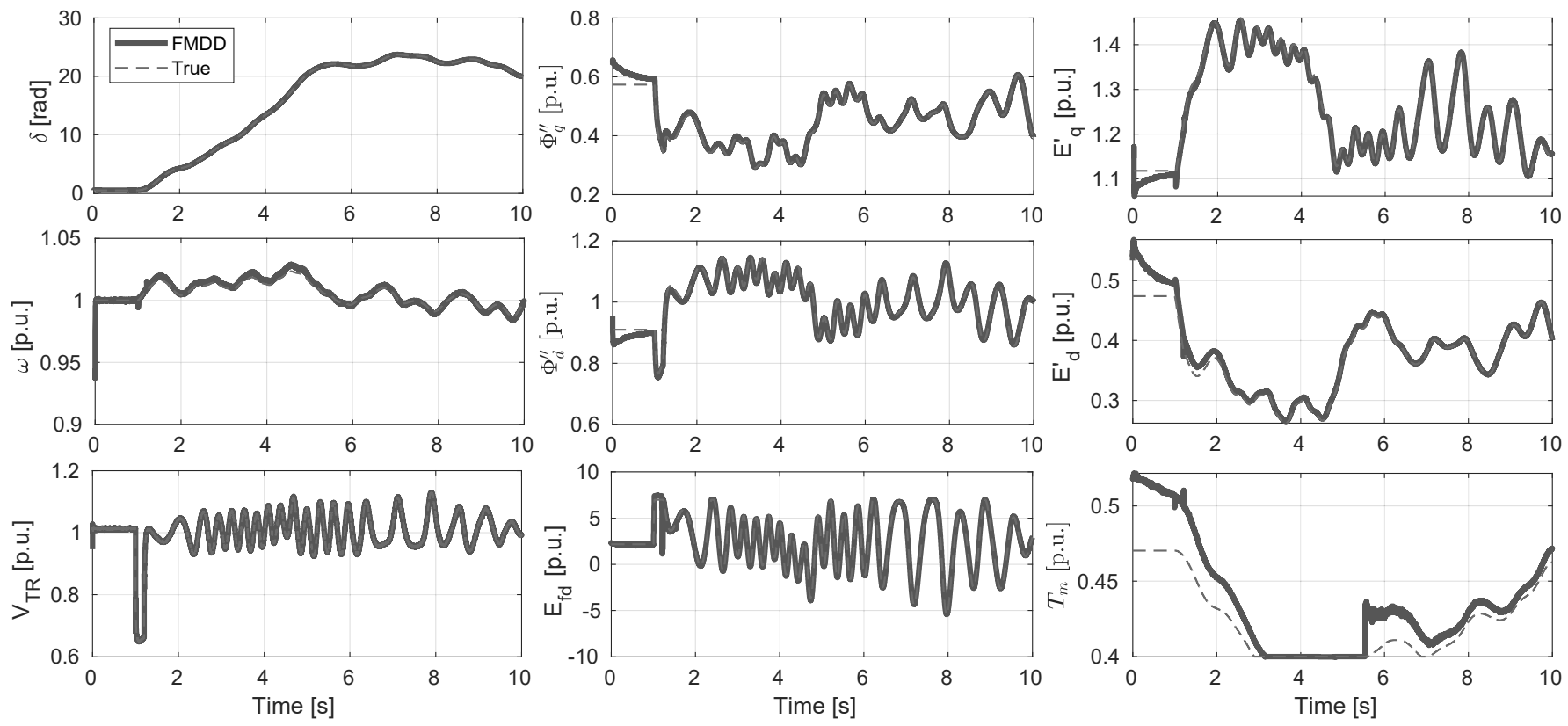

Fig. 16. Scenario D3: Global estimation of all the state variables of the generator G5 after a three-pase to ground fault with a clearing time of $200 \mathrm{~ms}$ occurs.

$$
\begin{gathered}
E_{d}^{\prime \prime}=E_{q}^{\prime} K_{7}+\Psi_{d}^{\prime \prime} K_{8} \\
E_{q}^{\prime \prime}=-E_{d}^{\prime} K_{9}-\Psi_{q}^{\prime \prime} K_{10} \\
T_{e}=E_{d}^{\prime \prime} I_{q}-E_{q}^{\prime \prime} I_{d} \\
I_{d}=I_{r e} \sin (\delta)-I_{i m} \cos (\delta) \\
I_{q}=I_{i m} \sin (\delta)+I_{r e} \cos (\delta) \\
S_{q}=\frac{E_{d}^{\prime \prime}}{\sqrt{E_{d}^{\prime \prime 2}+E_{q}^{\prime \prime 2}}} S_{f}\left(E_{d}^{\prime \prime}, E_{q}^{\prime \prime}, S_{1.0}, S_{1.2}\right) \\
S_{d}=\frac{E_{q}^{\prime \prime} K_{11}}{\sqrt{E_{d}^{\prime \prime 2}+E_{q}^{\prime \prime 2}}} S_{f}\left(E_{d}^{\prime \prime}, E_{q}^{\prime \prime}, S_{1.0}, S_{1.2}\right) . \\
K_{1}=x_{q}^{\prime}-x_{l s}, \quad K_{2}=x_{d}^{\prime}-x_{l s}, K_{3}=x_{d}-x_{d}^{\prime}, \\
K_{4}=\frac{x_{d}^{\prime}-x_{d}^{\prime \prime}}{\left(x_{d}^{\prime}-x_{l s}\right)^{2}}, K_{5}=x_{q}-x_{q}^{\prime}, K_{6}=\frac{x_{q}^{\prime}-x_{q}^{\prime \prime}}{\left(x_{q}^{\prime}-x_{l s}\right)^{2}}, \\
K_{7}=\frac{x_{d}^{\prime \prime}-x_{l s}}{x_{d}^{\prime}-x_{l s}}, \quad K_{8}=\frac{x_{d}^{\prime}-x_{d}^{\prime \prime}}{x_{d}^{\prime}-x_{l s}}, \\
K_{9}=\frac{x_{q}^{\prime \prime}-x_{l s}}{x_{q}^{\prime}-x_{l s}}, \quad K_{10}=\frac{x_{q}^{\prime}-x_{q}^{\prime \prime}}{x_{q}^{\prime}-x_{l s}}, K_{11}=\frac{\left(x_{q}-x_{l}\right)}{\left(x_{d}-x_{l}\right)}
\end{gathered}
$$

APPENDIX B

\section{NOMENCLATURE}

All the variables and constants used throughout this article are listed in Table III. They are assessed using the per-unit system, unless they are time constants, which are quantized in seconds, or phases, in radians.

\section{APPENDIX C \\ GENERATOR PARAmETERS}

The parameters used for the generator, excitation system and turbine governor are listed in Tables IV and V.

\section{REFERENCES}

[1] "IEEE guide for AC generator protection," IEEE Std C37.102-2006 (Revison of IEEE Std C37.102-1995), pp. 1-177, 2006.

[2] D. Reimert, Protective Relaying for Power Generation Systems. CRC Press, 2006

[3] R. D. Rana, R. P. Schulz, M. Heyeck, and T. R. Boyer, "Generator loss of field study for aep's rockport plant," IEEE Computer Applications in Power, vol. 3, no. 2, pp. 44-49, April 1990.

[4] C. Lee, L. Ma, C. Weng, and B. Chen, "Lessons learned from the generator loss of field at a cogeneration thermal power plant in Taiwan," IEEE Transactions on Power Systems, vol. 26, no. 4, pp. 2093-2100, Nov 2011.

[5] IEEE Power System Relaying Committee, "IEEE tutorial on the protection of synchronous generators," IEEE Power \& Energy Society, 2011.

[6] C. R. Mason, "A new loss-of-excitation relay for synchronous generators," Transactions of the American Institute of Electrical Engineers, vol. 68, no. 2, pp. 1240-1245, July 1949.

[7] J. Berdy, "Loss of excitation protection for modern synchronous generators," IEEE Transactions on Power Apparatus and Systems, vol. 94 no. 5, pp. 1457-1463, Sep. 1975.

[8] "SEL-700G, Generator and Intertie Protection Relays: Instruction Manual," Schweitzer Engineering Laboratories, INC, Tech. Rep., 2015.

[9] S. Protection and C. Subcommittee, "Power plant and transmission system protection coordination," Princeton, NJ, USA, Tech. Rep., 07 2010.

[10] J. A. Diaz de Leon and C. W. Taylor, "Understanding and solving shortterm voltage stability problems," in IEEE Power Engineering Society Summer Meeting, vol. 2, July 2002, pp. 745-752 vol.2.

[11] A. P. de Morais, G. Cardoso, and L. Mariotto, "An innovative loss-ofexcitation protection based on the fuzzy inference mechanism," IEEE Transactions on Power Delivery, vol. 25, no. 4, pp. 2197-2204, Oct 2010.

[12] T. Amraee, "Loss-of-field detection in synchronous generators using decision tree technique," IET Generation, Transmission Distribution, vol. 7, no. 9, pp. 943-954, Sep. 2013.

[13] A. M. Sharaf and T. T. Lie, "ANN based pattern classification of synchronous generator stability and loss of excitation," IEEE Transactions on Energy Conversion, vol. 9, no. 4, pp. 753-759, Dec 1994.

[14] M. Amini, M. Davarpanah, and M. Sanaye-Pasand, "A novel approach to detect the synchronous generator loss of excitation," IEEE Transactions on Power Delivery, vol. 30, no. 3, pp. 1429-1438, June 2015.

[15] A. Hasani and F. Haghjoo, "A secure and setting-free technique to detect loss of field in synchronous generators," IEEE Transactions on Energy Conversion, vol. 32, no. 4, pp. 1512-1522, Dec 2017. 
TABLE III

NOMENCLATURE USED FOR ALL VARIABLES AND CONSTANTS.

\begin{tabular}{|c|c|}
\hline$\delta / \omega$ & Rotor angle/angular velocity. \\
\hline$E_{d}^{\prime} / E_{q}^{\prime}$ & $\mathrm{d} / \mathrm{q}$ axis transient voltage. \\
\hline$\Psi_{d}^{\prime \prime} / \Psi_{q}^{\prime \prime}$ & d/q axis sub-transient flux. \\
\hline$E_{d}^{\prime \prime} / E_{q}^{\prime \prime}$ & $\mathrm{d} / \mathrm{q}$ axis equivalent voltage. \\
\hline$T_{e} / E_{f d}$ & Electric torque / Field voltage. \\
\hline$T_{m} / T_{m 0}$ & Instantaneous/Steady state mechanical torque. \\
\hline$I_{d} / I_{q}$ & d/q axis stator current. \\
\hline$V_{T R}$ & Transducer output signal. \\
\hline$V$ & Voltage magnitude at the point of connection. \\
\hline pso & Power system stabilizer signal. \\
\hline$S_{d} / S_{q}$ & d/q-axis saturation function. \\
\hline$S_{f}$ & Saturation non-linear function. \\
\hline$S_{1.0} / S_{1.2}$ & Saturation coefficients. \\
\hline$\omega_{0}$ & Nominal rotor speed (1 p.u.). \\
\hline$\omega_{s}$ & Angular nominal frequency. \\
\hline$r_{A} / x_{l s}$ & Stator resistance / Leakage reactance. \\
\hline$x_{d}, x_{q}$ & d/q axis synchronous reactances. \\
\hline$x_{d}^{\prime}, x_{q}^{\prime}$ & $\mathrm{d} / \mathrm{q}$ axis transient reactances. \\
\hline$x_{d}^{\prime \prime}, x_{q}^{\prime \prime}$ & d/q axis sub-transient reactances. \\
\hline$T_{d}^{\prime}, T_{q}^{\prime}$ & $\mathrm{d} / \mathrm{q}$ axis transient open circuit time constants. \\
\hline$T_{d}^{\prime \prime}, T_{q}^{\prime \prime}$ & $\mathrm{d} / \mathrm{q}$ axis sub-transient time constants. \\
\hline$D / H$ & Damping factor / Inertia constant. \\
\hline$K_{A} / \frac{1}{r}$ & Exciter / Turbine governor gain. \\
\hline$T_{R} / T_{A}$ & Transducer / Exciter time constant. \\
\hline$T_{e f}$ & Turbine governor effective time constant. \\
\hline$V_{R E F}$ & Reference voltage of the excitation system. \\
\hline$V_{R \max } / V_{R \min }$ & Max/min limits for the field voltage value. \\
\hline$T_{m \max } / T_{m \min }$ & Max/min limits for the mechanical torque. \\
\hline
\end{tabular}

TABLE IV

SYNCHRONOUS GENERATOR DATA IN PER UNIT $\left(S_{g e n}=900 \mathrm{MVA}\right)$. THE VARIABLES ARE IN P.U. AND THE TIME CONSTANTS IN SECONDS.

\begin{tabular}{|c|c|c|c|c|c|c|c|}
\hline$r_{a}$ & $x_{d}$ & $x_{d}^{\prime}$ & $x_{d}^{\prime \prime}$ & $T_{d o}^{\prime}$ & $T_{d o}^{\prime \prime}$ & $H$ & $D$ \\
\hline 0.0025 & 1.8 & 0.3 & 0.25 & 8 & 0.03 & 6.5 & 2 \\
\hline$x_{l}$ & $x_{q}$ & $x_{q}^{\prime}$ & $x_{q}^{\prime \prime}$ & $T_{q o}^{\prime}$ & $T_{q o}^{\prime \prime}$ & $S_{1.0}$ & $S_{1.2}$ \\
\hline 0.2 & 1.7 & 0.55 & 0.25 & 0.4 & 0.05 & 0.0654 & 0.5743 \\
\hline
\end{tabular}

TABLE V

EXCITATION AND TURBINE PARAMETERS. THE VARIABLES ARE IN P.U. AND THE TIME CONSTANTS IN SECONDS.

\begin{tabular}{|c|c|c|c|c|}
\hline$K_{a}$ & $T_{R}$ & $T_{A}$ & $V_{R \max }$ & $V_{R \min }$ \\
\hline 200 & 0.01 & 0.05 & 7 & -7 \\
\hline
\end{tabular}

\begin{tabular}{|c|c|c|c|c|c|c|c|}
\hline $1 / r$ & $T_{s}$ & $T_{c}$ & $T_{3}$ & $T_{4}$ & $T_{5}$ & $T_{m \max }$ & $T_{m \min }$ \\
\hline 25 & 0.1 & 0.5 & 0 & 1.25 & 5 & 0.4 & 1 \\
\hline
\end{tabular}

[16] M. Abedini, M. Sanaye-Pasand, and M. Davarpanah, "An analytical approach to detect generator loss of excitation based on internal voltage calculation," IEEE Transactions on Power Delivery, vol. 32, no. 5, pp. 2329-2338, Oct 2017.

[17] B. Mahamedi, J. G. Zhu, and S. M. Hashemi, "A setting-free approach to detecting loss of excitation in synchronous generators," IEEE Transactions on Power Delivery, vol. 31, no. 5, pp. 2270-2278, Oct 2016.

[18] I. Kiaei, S. Lotfifard, and A. Bose, "Secure loss of excitation detection method for synchronous generators during power swing conditions," IEEE Transactions on Energy Conversion, vol. 33, no. 4, pp. 19071916, Dec 2018.

[19] J. Zhao, A. Gómez-Expósito, M. Netto, L. Mili, A. Abur, V. Terzija, I. Kamwa, B. Pal, A. K. Singh, J. Qi, Z. Huang, and A. P. S. Meliopoulos, "Power system dynamic state estimation: Motivations, definitions, methodologies, and future work," IEEE Transactions on Power Systems, vol. 34, no. 4, pp. 3188-3198, July 2019.

[20] A. Rouhani and A. Abur, "A robust dynamic state estimator against exciter failures," in 2016 North American Power Symposium (NAPS), Sep. 2016, pp. 1-6.

[21] Y. Zhang and X. R. Li, "Detection and diagnosis of sensor and actuator failures using IMM estimator," IEEE Transactions on Aerospace and Electronic Systems, vol. 34, no. 4, pp. 1293-1313, Oct 1998.

[22] Z. Huang, P. Du, D. Kosterev, and S. Yang, "Generator dynamic model validation and parameter calibration using phasor measurements at the point of connection," IEEE Transactions on Power Systems, vol. 28, no. 2, pp. 1939-1949, May 2013.

[23] N. Zhou, D. Meng, Z. Huang, and G. Welch, "Dynamic state estimation of a synchronous machine using PMU data: A comparative study," IEEE Transactions on Smart Grid, vol. 6, no. 1, pp. 450-460, Jan 2015.

[24] E. Ghahremani and I. Kamwa, "Local and Wide-Area PMU-based decentralized dynamic state estimation in Multi-Machine power systems," IEEE Transactions on Power Systems, vol. 31, no. 1, pp. 547-562, Jan 2016.

[25] J. Zhao, M. Netto, and L. Mili, "A robust iterated extended Kalman filter for power system dynamic state estimation," IEEE Transactions on Power Systems, vol. 32, no. 4, pp. 3205-3216, July 2017.

[26] Siemens Energy INC, 400 State Street, PO Box 1058 Schenectady, NY 12301-1058 USA, "PSSE 32. 0. 5 Volume II Program Application Guide,” ISBN 1518-395-5000., October 2010.

[27] P. Sauer and M. Pai, Power System Dynamics and Stability. Stipes Publishing L.L.C., 2006.

[28] P. Marchi, F. Messina, L. Rey Vega, and C. G. Galarza, "Online tracking of sub-transient generator model variables using dynamic phasor measurements," Electric Power Systems Research, to be published.

[29] J. Zhao, L. Mili, and A. Gómez-Expósito, "Constrained robust unscented kalman filter for generalized dynamic state estimation," IEEE Transactions on Power Systems, vol. 34, no. 5, pp. 3637-3646, Sep. 2019.

[30] A. Hasani, F. Haghjoo, F. F. da Silva, and C. Leth Bak, "Synchronous generator loss of field protection: A real-time realistic framework and assessment of some recently proposed methods," IEEE Transactions on Power Delivery, vol. 34, no. 3, pp. 971-979, June 2019.

[31] J. C. K. W. Cheung and G. Rogers. Power system toolbox. [Online]. Available: http://www.eps.ee.kth.se/personal/vanfretti/ pst/Power_System_Toolbox_Webpage/PST.html

[32] H. G. Aghamolki, Z. Miao, L. Fan, W. Jiang, and D. Manjure, "Identification of synchronous generator model with frequency control using unscented kalman filter," Electric Power Systems Research, vol. 126, pp. 45 - 55, 2015. [Online]. Available: http://www.sciencedirect.com/science/article/pii/S0378779615001224

[33] NERC, "Technical reference document: Dynamic load modeling," North American Reliability Cooperation, Tech. Rep., 032016. 